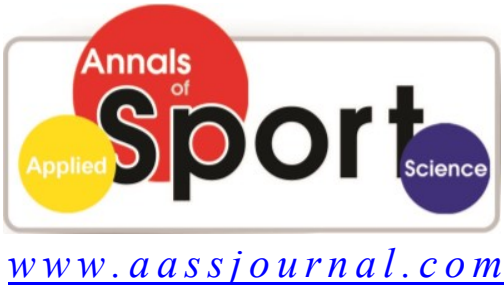

ISS N (Online): $2322-4479$

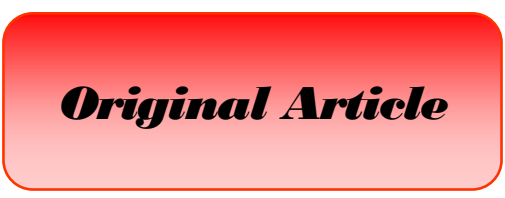

Received: $13 / 04 / 2014$

Accepted: 02/06/2014

\title{
The Effect of Oral Carbohydrate Solutions on the Performance of Swimmers
}

\section{1,2 Laleh Afshari, ${ }^{2,3}$ Shooka Mohammadi*, ${ }^{4}$ Saeid Shakerian, ${ }^{1,2}$ Reza Amani}

1. Department of Nutritional Science, Arvand International Division, Ahvaz Jundishapur University of Medical Sciences, Abadan, Iran.

2. Department of Nutrition and Dietetics, Faculty of Para Medicine, Ahvaz Jundishapur University of Medical Sciences, Ahvaz, Iran.

3. Dietetics and Nutritional Sciences Programmes, School of Healthcare Sciences, Faculty of Health Sciences, Universiti Kebangsaan Malaysia, Kuala Lumpur, Malaysia.

4. Department of Physical Education, Shahid Chamran University of Ahvaz, Ahvaz, Iran.

\section{ABSTRACT}

It is well established that carbohydrate solutions can improve the performance in prolonged exercise. The aim of this study was to compare the impact of sugar and glucose solutions on exercise performance of swimmers. Twelve male teenager elite Iranian swimmers aged 12-17 years from Waterpolo Team of Ahvaz Oil Industry participated in a double-blind cross-over trial. They consumed three oral $6 \%$ purified carbohydrate solutions as glucose, sugar or placebo (aspartame) formulas in three non-consecutive days. In each day the swimmers undertook a $2 \times 200$-meter incremental swimming by 15 minutes time interval. Before starting the second course, subjects consumed their solutions. Blood glucose levels and time elapsed in two phases were recorded. Longer Swimming time significantly caused by sugar solution in the second course. Blood glucose level was increased by sugar and glucose solutions higher than the placebo before starting the second swim $(\mathrm{p}<0.05)$. However, after swimming, blood glucose concentrations were significantly elevated in all groups. After drinking a sugar solution and before starting the second 200-m swimming, the blood glucose level was higher than two other groups at this phase. Oral 6\% sugar solution increased the time of swimming compared with oral glucose and placebo solutions in a $200-\mathrm{m}$ swim. It can be explained by differences in Glycemic index in which sucrose has a lower GI than glucose.

Key Words: Glucose Solution, Swimming Time, Blood Glucose. 


\section{INTRODUCTION}

Sports drinks consist of components which provide energy, essential nutrients and the balance of body fluids (1). It is a common practice for athletes from different sports to ingest carbohydrate-electrolyte solutions for performance purposes. The ergogenic effects of carbohydrate intake during prolonged exercise have been well explained (2).

Glucose availability and an increase in peripheral glucose requirements during prolonged exercise has been highlighted (3). In order to maximize prolonged endurance performance, it is suggested that athletes should have $\mathrm{CHO}$ intake during endurance activities (2). Carbohydrates are one of the two main fuels for optimal sport performances (4). Ingestion of carbohydrate is a general contributor and ergogenic agents to fulfill daily energy needs of athletes during a sports event or during the days preceding it $(1,4,5)$.

The main determinants of substrate utilization during exercise are the strength and the length of the exercise as well as the pre-exercise carbohydrate stores and the training status of the athlete (6). Ingestion of $\mathrm{CHO}$ from 15 to $20 \mathrm{~min}$ prior to the exercise results in an increase in plasma glucose (7), the time of performance (8), $\mathrm{CHO}$ oxidation during exercise (9), and reduces endogenous glucose production (10).

The $\mathrm{CHO}$ can be in the forms of maltodextrins, glucose, sucrose, or some high glycemic starches. Fructose should be limited for the reason of the likelihood of gastrointestinal distress. The rate of $\mathrm{CHO}$ ingestion can be along with drinking 600$1200 \mathrm{ml} / \mathrm{h}$ of a solution containing $4-8 \% \mathrm{CHO}$ (4-8 g/100 ml) (11). CHO solutions containing $6-8 \%$ will increase time trial performance $(\sim 2 \%)$ and time to exhaustion $(\sim 15 \%)$. More than $8-10 \%$ solutions can enhance endurance exercise in events which are longer 1 hour $(4,12)$. The rate of glycogen resynthesis depends on the amount of $\mathrm{CHO}$ ingested (13). Sport drinks which contain 6\% $(\mathrm{w} / \mathrm{v})$ glucose solution are effective for exercise performances (14). Carbohydrate ingestion during long-duration exercise, lasting 2 hours or more, almost always delays the onset of fatigue and improves the performance. However, ingesting too much carbohydrate can have detrimental effects (15). Sports beverages containing CHOs and electrolytes may be consumed before, during and after the exercises to help maintain blood glucose concentration, provide fuel for muscles, and decrease the risk of dehydration and hyponatremia.

The Glycemic Index (GI) categorizes CHO-rich foods based on their postprandial blood glucose response compared with a reference food. Sugar (Sucrose), as a disaccharide, consists of $50 \%$ glucose and $50 \%$ fructose, while it has a glycemic index in a similar amount lower than glucose. Sucrose is absorbed quickly, but it has a low glycemic index because of containing of fructose which has a slight influence on blood glucose (16). Basically, the GI aimed to point out the total rate of digestion and absorption of the $\mathrm{CHO}$ in a food. If the GI of $\mathrm{CHO}$ causes the rate of blood glucose to response to $\mathrm{CHO}$, it seems reasonable that ingestion of $\mathrm{CHO}$ with different GI before, during and after exercise will impact sport performance. However, there is still much uncertainty and an insufficiency of data about the profits of ingestion of high GI and low GI $\mathrm{CHO}$ to enhance performance (17).

Endurance sports are rising in popularity, and athletes are seeking ways to enhance their performance at all levels by training and nutrition. For endurance exercises with duration of $30 \mathrm{~min}$ or more, the most likely causes of fatigue are dehydration and carbohydrate reduction. Recently, the role of carbohydrate intake has been established to improve performance during shorter lengths and of higher intensity exercise (2). It is thought that during higher intensity exercise, 
plasma glucose concentrations do not decrease, as there is sufficient glycogen to sustain the short duration of exercise ( $\sim 60$ min) (6). To our knowledge, no studies have proven that consuming large amounts of $\mathrm{CHO}$ will cause higher exogenous $\mathrm{CHO}$ oxidation rates and improvement of performance. Until recently, the positive effects of carbohydrate feeding were only demonstrated with exercises lasting over two hours in duration (15). Hence, the present investigation aimed to determine the effects of glucose formula on short duration exercise and sport performance of swimmers in comparison to sugar and aspartame solutions.

\section{MATERIALS AND METHODS}

Participants. Twelve healthy male elite swimmers in the age range of 12-17 years from waterpolo team of Ahvaz oil industry participated in this double-blind cross-over trial. The study was approved by the ethical committee of Ahvaz Jondishapur University of Medical Sciences. The volunteers were briefed on the purpose of the study, experimental protocol and their written consents were obtained.

Liquid Supplements. The carbohydrate solution used in the present study was prepared by researchers. In order to prepare $6 \%$ glucose, 6\% sugar and aspartame solutions, $6 \mathrm{gm}$ of them was dissolved in 100 $\mathrm{ml}$ of water separately. The placebo solution was matched in formulation to the carbohydrate solution except that it did not contain $\mathrm{CHO}$ and contained artificial sweeteners (aspartame).

Procedure. Twelve male teenage elite swimmers consumed three oral $6 \%$ purified carbohydrate solutions $(6 \mathrm{~g} / 100 \mathrm{ml})$ as glucose, sugar or a placebo (aspartame) formula in three non-consecutive days. During each day, the swimmers tried two 200 meters course separated by 15 minutes between each course. Immediately after finishing the first course, subjects consumed their solutions. Blood glucose levels and time elapsed in two phases were recorded. The reason for deferral carbohydrate feeding until $5-15 \mathrm{~min}$ before starting an activity is to minimize the glycemic and insulinaemic responses during exercise (18).

In the three separate ingestion trials, all swimmers ingested the equivalent of $200 \mathrm{ml}$ of a $6 \%$ of sugar $(19,20)$, glucose and matched placebo solution in 15-min intervals before the second swim in three nonconsecutive days separately. The solutions were served in two plastic volumetric syringes (Kendall Monoject, Mansfield, MA). The solutions were weighed using an electronic balance (Mettler, Toledo AB54-s, Greifensee, Switzerland) to ensure the correct volume was ingested $15 \mathrm{~min}$ before the exercise. A container contains the solution which was placed on the same electronic balance to ensure that the correct volume of the solution was taken up into the plastic syringe. The feeding schedule was designed to provide the swim with approximately $6 \mathrm{~g} \mathrm{CHO}$.

Statistical Analysis. Statistical Package for Social Sciences (SPSS) version 20 was used for the analysis. The mean was applied for all the variables. Paired t-test was used to compare the performance time and blood sugar level between the first and the second swim among three groups. Repeated measures ANOVA analysis was applied to study the difference before and after trial. Differences were considered significant at $\mathrm{P}<0.05$.

\section{RESULTS}

The mean blood sugar and performance time of the subjects showed no significant difference between the trials using repeatedmeasures ANOVA. The ANOVA results for blood sugar and performance time were $[\mathrm{F}(2$, $33)=0.053 ; \mathrm{P}>0.05]$ and $[\mathrm{F}(2,33)=0.02$; $\mathrm{P}>0.05]$ respectively. The subjects were not aware of the drinks consumed during trials. Oral 6\% sugar solution increased the time of swimming compared with oral glucose and 
placebo solutions in $200 \mathrm{~m}$ course (Figure 2). The mean total exercise time showed significant difference between two swims in the sugar group (Table 1).

During exercise the blood glucose levels showed a significant increase $(\mathrm{P}<0.05)$ with an increase after the first swim and before the second swim in sugar and glucose group $(\mathrm{P}<0.05)$ (Table 1). The result of paired t-test for post exercise blood glucose levels of the subjects in all the three trials showed a significant increase after the second swim $(\mathrm{P}<0.05)$ (Table 1). The trials with glucose and sugar recorded no significantly higher blood glucose levels when compared with aspartame. The trend of blood glucose in subjects before and after the first and the second swims is shown in the Figure1. However, in each time interval no significant difference was observed in the blood glucose levels between the trials.

Table 1. comparison of the time of performance and blood sugar between first and second swim among three groups

\begin{tabular}{lccc}
\hline Trials & $\begin{array}{c}\text { Sugar } \\
(\mathbf{n = 1 2})\end{array}$ & $\begin{array}{c}\text { Glucose } \\
(\mathbf{n = 1 2})\end{array}$ & $\begin{array}{c}\text { Placebo(Aspartam) } \\
(\mathbf{n}=12)\end{array}$ \\
\hline Mean blood sugar before first swim (mg/dl) & 87.6 & 89.3 & 89.6 \\
Mean blood sugar before second swim (mg/dl) & 99.5 & 96.5 & 96.6 \\
P-Value & $0.002^{*}$ & $0.006^{*}$ & $0.110^{\mathrm{NS}}$ \\
& & & \\
Mean blood sugar before second swim (mg/dl) & 99.5 & 96.5 & 96.6 \\
Mean blood sugar after second swim (mg/dl) & 108.3 & 105.8 & 105.1 \\
P-Value & $0.008^{*}$ & $0.025^{*}$ & $0.005^{*}$ \\
& & & \\
Mean of time for first swim (min: s: ss) & $2: 57: 81$ & $2: 57: 62$ & $2: 58: 60$ \\
Mean of time for second swim(min:s:ss) & $3: 1: 76$ & $2: 58: 87$ & $2: 59: 61$ \\
P-Value & $0.017^{*}$ & $0.323^{\mathrm{NS}}$ & $0.496^{\mathrm{NS}}$ \\
\hline
\end{tabular}

* Significant at $P<0.05$; NS: Not significant

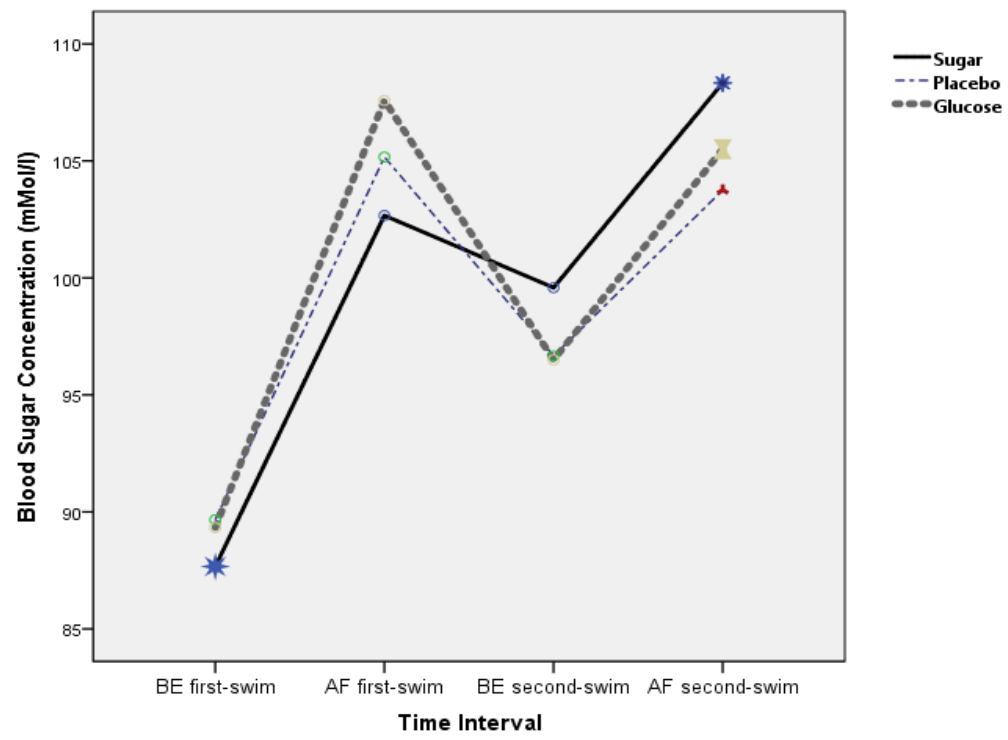

Fig. 1. Repeated measures ANOVA. Blood sugar level of the subjects before and after first and second swims among three groups (Sugar;Glucose; Placebo) $(\mathrm{n}=12)$ 


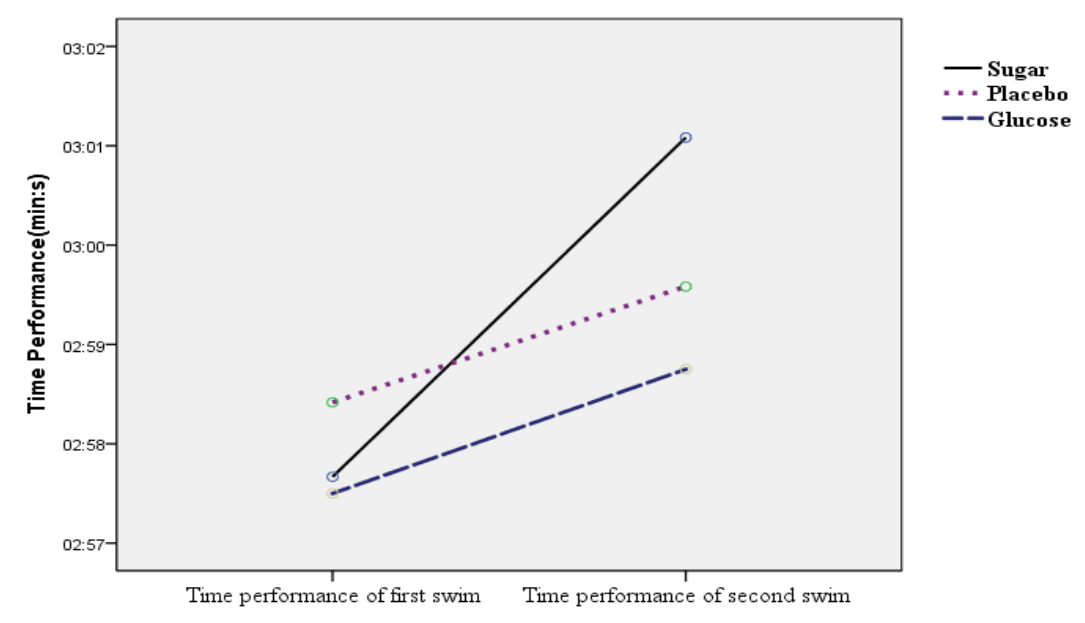

Fig. 2. Performance time of the subjects for first and second swims among three groups (Sugar; ;Glucose; Placebo) $(\mathrm{n}=12)$

\section{DISCUSSION}

The aim of this study was to compare the effect of sugar and glucose solutions on exercise performance of swimmers. The main finding of the study was that ingestion of sugar solution significantly increased the time of swimming performance in comparison with the same volume glucose or placebo solutions. Furthermore, relative sucrose has a lower GI than glucose and glucose consumption might be predictable to stimulate greater muscle glycogen resynthesis than sucrose. However, due to its fructose content, sucrose may favorably renovate liver, rather than muscle glycogen stores post exercise (21).

Even though it has been reported in a study in the UK (21) that there was not any significant differences in mean exercise time among subjects through ingestion of sugar and glucose solutions. The researchers demonstrated that highly-trained subjects by administration of glucose were capable to do exercise for longer while fasting without any further decrease in muscle glycogen. They suggested that after the end of exercise, oxidation of $\mathrm{CHO}$ was retained as a result of glucose ingestion, which was adequate to equalize the reduction in muscle glycogen oxidation that happened as a consequence of glycogen depletion, allowing exercise to continue. The reduction of blood glucose, and subsequent decrease in muscle glucose uptake and oxidation during the final stages of exercise without $\mathrm{CHO}$ ingestion, was believed to be a main source of fatigue (21).

It has to be mentioned that carbohydrate ingestion before exercise can cause hyperglycemia and hyperinsulinaemia following by a rapid reduction in blood glucose 15-30 min after exercise, changed to reactive hypoglycemia (14). The decline in blood glucose is most likely the consequence of an increased muscle glucose uptake and reduced liver glucose output. Furthermore, hyperinsulinaemia following by carbohydrate ingestion inhibits lipolysis and fat oxidation and this may result in quick muscle glycogen depletion. Therefore, pre-exercise carbohydrate ingestion may have the potential to impair performance. However, only two studies (22, 23) have reported reduced performance 
ability, whereas the majority of studies have stated no changes or an enhancement in performance following pre-exercise carbohydrate ingestion (24).

Ingestion of $\mathrm{CHO}$ during endurance exercise will lead to keep the blood glucose levels, related increase in the rate of $\mathrm{CHO}$ oxidation and release glycogen of muscle during the last stages of the exercise (25). During first and second swim, the blood sugar values increased in sugar, glucose and placebo trials and no significant differences were found between the trials. This increase in glucose concentrations with $\mathrm{CHO}$ solutions in this study was also similar to the responses found in study conducted by de Salles Painelli, Nicastro, and Lancha (26) which showed that solution with $6.4 \%$ glucose or maltodextrin improved around $2-3 \%$ of the time trial and average power in comparison with placebo. No differences have been found among the solutions with different type of $\mathrm{CHO}$.

Of course, the performance properties and metabolism of carbohydrate ingestion just before exercise (5-15 min) look like the ingestion of them during the activity. In addition, a rebound hypoglycemia in the initial phase of exercise seems not to influence exercise performance (24). This implies that there is no need to escape carbohydrate intake pre exercise. However, carbohydrate ingestion during exercise has also been proven to increase exercise performance even in high intensity exercise and relatively short duration, and it has become clear that mechanisms for the effect during this type of exercises is not metabolic, but it is through the central nervous system (27). There is different instruction for different endurance sports. Carbohydrate ingestion results in improved performance in cycling and running, although the mechanism may not necessarily be the same (28).

Although in another study (29) it was reported that exercise performances improved with mouth rinse, Whitham and McKinney (30) did not find the same outcome of $6 \%$ maltodextrin solution in a running procedure. One possible reason for differences between the results from Rollo and Williams (31) and Whitham and McKinney (30) depends on nutritional status of the runners in each study. While the subjects of Rollo's study went to the laboratory after an overnight fasting, standardization of the subjects' diet has been done in the study by Whitham and McKinney and they requested athletes to drink it 4 hours prior the trial.

In contrast to the studies that have reported improvements in cycling time trial performance of fasted cyclists after mouth rinsing with a carbohydrate solution $(27,32$, $33)$, it has been reported by Beelen et al. (34) that there was no improvement in cycling performance when the cyclists were fed rather than faceted. Also in the current study, there were no differences in the time of performances between the carbohydrate solutions and placebo trials. Consequently, it is hard to evaluate the potential effectiveness of glucose ingestion due to the significant effect only under conditions where the liver's glycogen may be depleted.

A cycling study reported that the improvement of endurance capacity when fluid was ingested maybe a result of heart rate reduction, core temperature and utilization of muscle glycogen in comparison with cycling without fluid ingestion (35). Ingesting (31) and mouth rinsing $\mathrm{CHO}$ can independently improve endurance performance $(29,33)$. However, the potential performance benefit of ingesting carbohydrate solutions appears to be influenced by the $\mathrm{CHO}$ status of the participant before exercise.

It is possible to assume that feeding before exercise may have an impact on the brain responses to an oral $\mathrm{CHO}$ stimulus during exercise because it activates the brain regions associated with feeding and reward and is controlled by the physiological status of the body a homeostatic regulation (36). In this context, it can be supposed that the 
nutritional status has a direct impact on the ergogenic effectiveness of $\mathrm{CHO}$ solutions.

\section{CONCLUSION}

Oral 6\%-sugar solution increased the time of swimming compared with oral glucose and placebo solutions in the 200-m course. Overally, this study indicates that there is a difference in glycemic index between glucose and sugar (sucrose), and therefore blood glucose availability may have important consequences for subsequent exercise capacity. So far, no studies have examined the relative advantages of glucose and sucrose administration, during recovery from swimming, in terms of subsequent endurance exercise capacity. Based on the available indications, it appears to be no reason not to ingest glucose before exercise as it does not appear to have negative effects on performances. Athletes who have symptoms that are often associated with reactive hypoglycemia can find solutions to avoid it. These solutions could include choosing low GI carbohydrates, ingesting carbohydrate just preexercise or during a warm-up or alternatively, avoiding carbohydrate in $90 \mathrm{~min}$ before exercise altogether (28). In addition, $\mathrm{CHO}$ solutions may have an impact on the mechanisms of fatigue that has not been evaluated among swimmers yet. However, more controlled studies on the actual role of oral $\mathrm{CHO}$ solutions in different nutritional status are required. Furthermore, further studies should evaluate if the same trial could improve exercise performance in other sports.

\section{ACKNOWLEDGMENTS}

The source of data used in this paper was from a bachelor thesis by Laleh Afshari, Master student of Arvand International Division of Ahvaz Jundishapur University of Medical Sciences, and financial support was provided by Ahvaz Jundishapur University of Medical Sciences.

This work was supported by Ahvaz Jundishapur University of Medical Sciences. (Grant number 87s.3). This study was approved by the Ethics of Ahvaz Jundishapur University of Medical Sciences, Ahvaz, Iran, and registered in the Iranian Registry of Clinical Trials (IRCT number IRCT2014031716910N1), Conflict of Interest: None declared.

\section{REFFRENCES}

1. Karelis AD, Smith JW, Passe DH, Peronnet F. Carbohydrate administration and exercise performance: what are the potential mechanisms involved? Sports medicine (Auckland, NZ). 2010;40(9):747-63.

2. Jeukendrup AE. Carbohydrate feeding during exercise. European Journal of Sport Science. 2008;8(2):77-86.

3. Lieberman HR, Falco CM, Slade SS. Carbohydrate administration during a day of sustained aerobic activity improves vigilance, as assessed by a novel ambulatory monitoring device, and mood. The American journal of clinical nutrition. 2002;76(1):120-7.

4. Rodriguez NR, Di Marco NM, Langley S. American College of Sports Medicine position stand. Nutrition and athletic performance. Medicine and science in sports and exercise. 2009;41(3):709-31.

5. Burke LM, Hawley JA, Wong SH, Jeukendrup AE. Carbohydrates for training and competition. Journal of sports sciences. 2011;29 Suppl 1:S17-27.

6. Jeukendrup AE. Carbohydrate and exercise performance: the role of multiple transportable carbohydrates. Current opinion in clinical nutrition and metabolic care. 2010;13(4):452-7.

7. Angus DJ, Hargreaves M, Dancey J, Febbraio MA. Effect of carbohydrate or carbohydrate plus medium-chain triglyceride ingestion on cycling time trial performance. Journal of applied physiology (Bethesda, Md : 1985). 2000;88(1):113-9.

8. Welsh RS, Davis JM, Burke JR, Williams HG. Carbohydrates and physical/mental performance during intermittent exercise to fatigue. Medicine and science in sports and exercise. 2002;34(4):723-31.

9. Arkinstall MJ, Bruce CR, Nikolopoulos V, Garnham AP, Hawley JA. Effect of carbohydrate ingestion on metabolism during running and cycling. Journal of applied physiology (Bethesda, Md : 1985). 2001;91(5):2125-34 
10. McConell GK, Canny BJ, Daddo MC, Nance MJ, Snow RJ. Effect of carbohydrate ingestion on glucose kinetics and muscle metabolism during intense endurance exercise. Journal of applied physiology (Bethesda, Md : 1985). 2000;89(5):1690-8.

11. Casa DJ, Armstrong LE, Hillman SK, Montain SJ, Reiff RV, Rich BS, et al. National athletic trainers' association position statement: fluid replacement for athletes. Journal of athletic training. 2000;35(2):212-24.

12. Temesi J, Johnson NA, Raymond J, Burdon CA, O'Connor HT. Carbohydrate ingestion during endurance exercise improves performance in adults. The Journal of nutrition. 2011;141(5):890-7.

13. Cermak NM, van Loon LJ. The use of carbohydrates during exercise as an ergogenic aid. Sports medicine (Auckland, NZ). 2013;43(11):1139-55.

14. Jeukendrup AE. Carbohydrate intake during exercise and performance. Nutrition (Burbank, Los Angeles County, Calif). 2004;20(7-8):669-77.

15. Jeukendrup A. Carbohydrate Supplementation during Exercise: Does It Help? How Much is Too Much? Sports Science Exchange. 2007;20(3):1-6.

16. Foster-Powell K, Holt SH, Brand-Miller JC. International table of glycemic index and glycemic load values: 2002. The American journal of clinical nutrition. 2002;76(1):5-56.

17. Donaldson CM, Perry TL, Rose MC. Glycemic index and endurance performance. International journal of sport nutrition and exercise metabolism. 2010;20(2):154-65.

18. Moseley L, Lancaster GI, Jeukendrup AE. Effects of timing of pre-exercise ingestion of carbohydrate on subsequent metabolism and cycling performance. European journal of applied physiology. 2003;88(4-5):453-8.

19. Angus DJ, Febbraio MA, Lasini D, Hargreaves M. Effect of carbohydrate ingestion on glucose kinetics during exercise in the heat. Journal of applied physiology (Bethesda, Md : 1985). 2001;90(2):601-5.

20. Wallis GA, Dawson R, Achten J, Webber J, Jeukendrup AE. Metabolic response to carbohydrate ingestion during exercise in males and females. American journal of physiology Endocrinology and metabolism. 2006;290(4):E708-15.

21. Casey A, Mann R, Banister K, Fox J, Morris PG, Macdonald IA, et al. Effect of carbohydrate ingestion on glycogen resynthesis in human liver and skeletal muscle, measured by (13)C MRS. American journal of physiology Endocrinology and metabolism. 2000;278(1):E65-75.

22. Foster C, Costill DL, Fink WJ. Effects of preexercise feedings on endurance performance. Medicine and science in sports. 1979;11(1):1-5.

23. Koivisto VA, Karonen SL, Nikkila EA. Carbohydrate ingestion before exercise: comparison of glucose, fructose, and sweet placebo. Journal of applied physiology: respiratory, environmental and exercise physiology. 1981;51(4):783-7.

24. Jeukendrup AE, Killer SC. The myths surrounding pre-exercise carbohydrate feeding. Annals of nutrition \& metabolism. 2010;57 Suppl 2:18-25.

25. Christ-Roberts CY, Mandarino LJ. Glycogen synthase: key effect of exercise on insulin action. Exercise and sport sciences reviews. 2004;32(3):90-4.

26. de Salles Painelli V, Nicastro H, Lancha AH, Jr. Carbohydrate mouth rinse: does it improve endurance exercise performance? Nutrition journal. 2010;9:33.

27. Chambers ES, Bridge MW, Jones DA. Carbohydrate sensing in the human mouth: effects on exercise performance and brain activity. The Journal of physiology. 2009;587(Pt 8):1779-94.

28. Jeukendrup AE. Nutrition for endurance sports: marathon, triathlon, and road cycling. Journal of sports sciences. 2011;29 Suppl 1:S91-9.

29. Rollo I, Cole M, Miller R, Williams C. Influence of mouth rinsing a carbohydrate solution on 1-h running performance. Medicine and science in sports and exercise. 2010;42(4):798-804.

30. Whitham M, McKinney J. Effect of a carbohydrate mouthwash on running time-trial performance. Journal of sports sciences. 2007;25(12):1385-92.

31. Rollo I, Williams C. Influence of ingesting a carbohydrate-electrolyte solution before and during a 1-hr running performance test. International journal of sport nutrition and exercise metabolism. 2009;19(6):645-58.

32. Carter JM, Jeukendrup AE, Jones DA. The effect of carbohydrate mouth rinse on 1-h cycle time trial performance. Medicine and science in sports and exercise. 2004;36(12):2107-11.

33. Pottier A, Bouckaert J, Gilis W, Roels T, Derave W. Mouth rinse but not ingestion of a carbohydrate solution improves 1-h cycle time trial performance. Scandinavian journal of medicine \& science in sports. 2010;20(1):105-11.

34. Beelen M, Berghuis J, Bonaparte B, Ballak SB, Jeukendrup AE, van Loon LJ. Carbohydrate mouth rinsing in the fed state: lack of enhancement of time-trial performance. International journal of sport nutrition and exercise metabolism. 2009;19(4):400-9.

35. Winnick JJ, Davis JM, Welsh RS, Carmichael MD, Murphy EA, Blackmon JA. Carbohydrate feedings during team sport exercise preserve physical and CNS function. Medicine and science in sports and exercise. 2005;37(2):306-15.

36. Small DM, Zatorre RJ, Dagher A, Evans AC, Jones-Gotman M. Changes in brain activity related to eating chocolate: from pleasure to aversion. Brain : a journal of neurology. 2001;124(Pt 9):1720-33. 


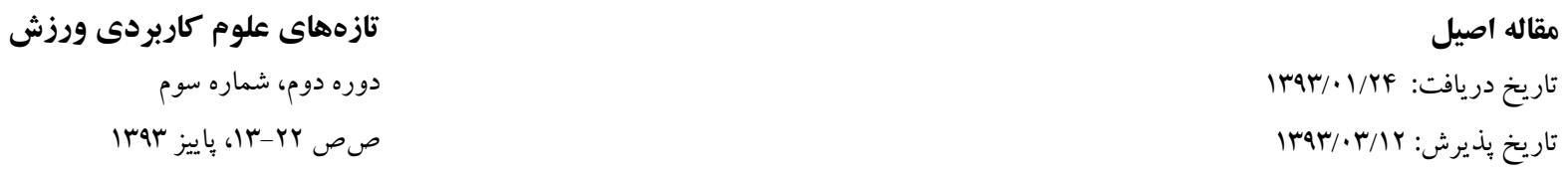

\section{اثر مصرف محكلول هاى قنلدى خوراكى بر عملكرو شناتَران

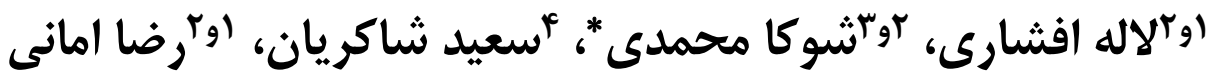

ا. كروه تغذيه، واحد بين الملل اروند آبادان، دانشگاه علوم يزشكى جندى شايور اهواز، آبادان، ايران.

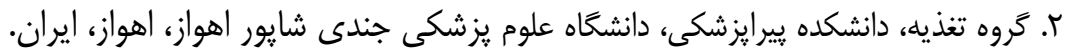

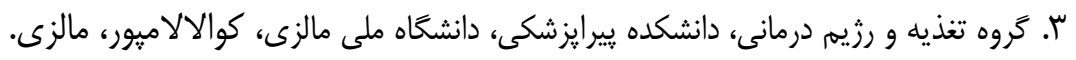

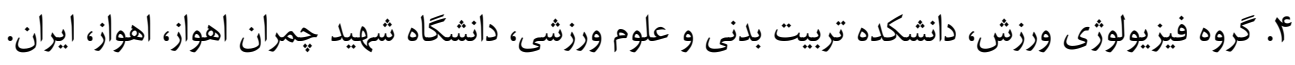

جكيده

مشخص شده است كه محلول هاى قندى مى تواند عملكرد ورزشكار را در ورزش طولانى مدت بهبود ببخشد. هدف از اين مطالعه مقايسه اثر

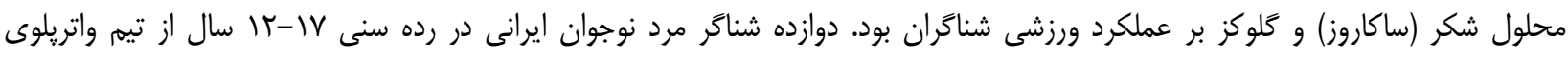

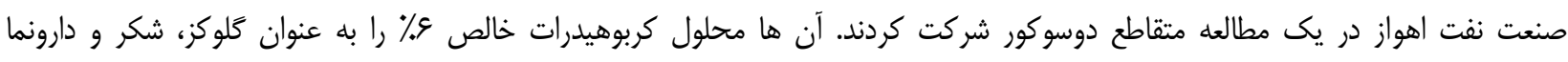

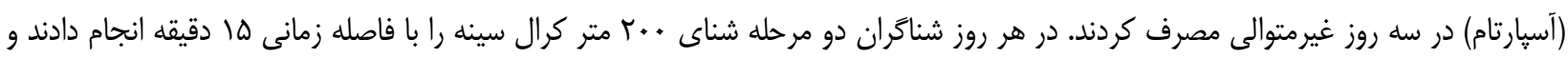

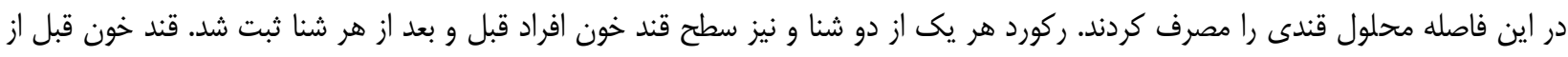

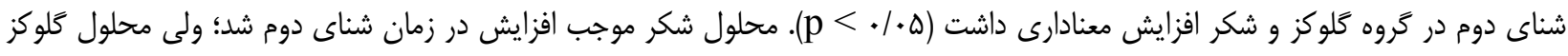

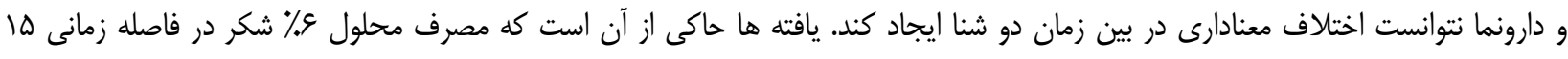

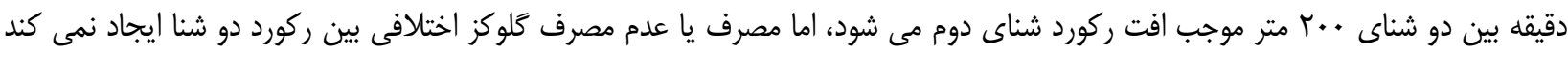

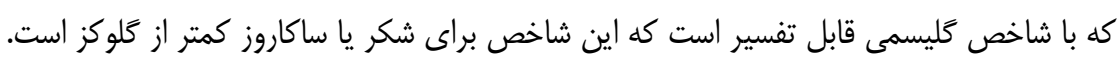
وازَّان كليدى: محلول كلوكز، زمان شنا، قند خون، زمان اجرا.

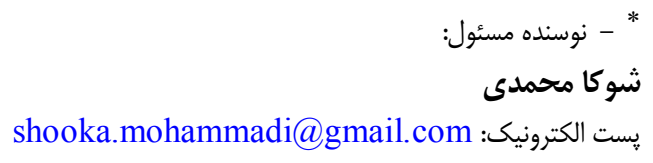


